\title{
A Survey on Visualization Techniques to Narrate Interpersonal Interactions between Sportsmen
}

\author{
Zainal Abidin \\ Sekolah Teknik Elektro dan Informatika \\ Institute Teknologi Bandung \\ Bandung, Indonesia \\ zainal@ti.uin-malang.ac.id
}

\author{
Dwi H Widyantoro \\ Sekolah Teknik Elektro dan Informatika \\ Institute Teknologi Bandung \\ Bandung, Indonesia \\ dwi@stei.itb.ac.id
}

\author{
Saiful Akbar \\ Sekolah Teknik Elektro dan Informatika \\ Institute Teknologi Bandung \\ Bandung, Indonesia \\ saiful@informatika.org
}

\begin{abstract}
Technological advances have resulted in rapid growth in personal activity data documentation. Chronological activity data flowing can narrate a story. Writing a story needs the means to visualize interactions to know the relationships between characters. This article explores time-oriented data visualization techniques. Exploration aims to investigate visualizations that might be used to narrate activities about interpersonal interactions. We map data visualizations based on completeness of story elements and shape flexibility. Based on the analysis of visualization techniques, we are looking for a flow visualization technique from events that can describe the event in detail.
\end{abstract}

Keywords-visualization, storytelling, interaction, chart, diagram

\section{INTRODUCTION}

The activities of athletes are publicly available on the website, such as on a personal website, organization, or federation. Activity athletes tend to interact with other athletes, especially when in a race or competition. In general, interactions between athletes are displayed in chronological tables. The appearance of the table cannot generate intrinsic meaning from the data [1]. The reader cannot capture the story from behind the presentation of interaction data in the table [2]. Bar and line charts have been used to visualize data in chronological order. Priestley first introduced historical visualization in chronological space in the late 17th century [3]. Priestley has begun the use of bar and line charts to narrate the history of the journey of a character, nation, or state. Priestley can describe quantity chronologically. The bar chart and the timeline do not inform the relationship between athletes.

Chronologically arranged athlete activities can be narrated into the career story of an athlete. The author collects and analyses athlete data, which is the subject of writing. The writer needs to know who the characters are involved in, their actions, and the results of each interaction.

DAM d. 3074 B C 930
Seth d. 2962. 912
Cainand. 2769. 910
Mahalaleel d. 2714. 895
Jared d. 2582. 962
Enoch d. 3017.365
Methufelah d. $2349 \cdot 969$
Lamech d. 2353.777
Noah d. 1998. 950
Shem d. 1846. 600

Fig. 2 : Catalogue as a source of biographical chart (reprint from [3])
Therefore, the writer needs the media to analyse the relationship between interactions in detail.

Charts and diagrams have been developed to visualize a story or history [2]. However, visualization tends to be aimed at analysing story content, not suitable for means of writing historical narratives. Writing history requires the media to display the facts of events, the characters involved, and their relationships [4]. This paper investigates techniques for visualizing time-oriented data flow. We collected several charts and diagrams that were used to illustrate a storyline. We group them into two categories. The plot visualization category uses charts and diagrams. We find diagrams or charts that can be used to visualize data as a narrative of a story.

\section{RELATED WORK}

Visualization is displaying a process or act into visible form [5]. Data visualization began to grow at the end of the 14th century [6]. Visualization of time-oriented data has been examined from various perspectives. Silva and Catarci examined the technique of displaying data oriented to the data visualization technique [7]. Silva and Catarci examine visualization techniques from the perspective of data visualization techniques and navigation as a facility for interacting with applications.

Seal and Heer discuss visualization techniques to tell data in the fields of journalism and education [1]. Exploration emphasizes the elements of data visualization and interaction to tell data. The analysis succeeded in mapping it to magazine type visual media, associated grap, partitioned poster, flow chart, comic strip, slide show, and video.

Thudt et al. examined the subjectivity of the narrator in the selection of data for visualization [8]. The focus of the study is on the visualization of personal data. Exploration succeeded in showing the position of the narrator's

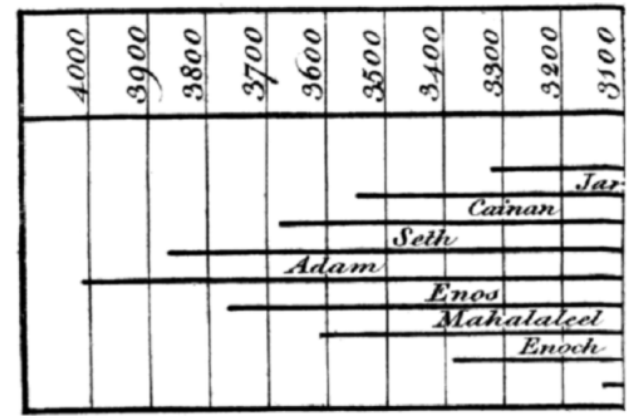

Fig. 1 : Priesley Chart for Hebrew succession (reprint from [3]) 


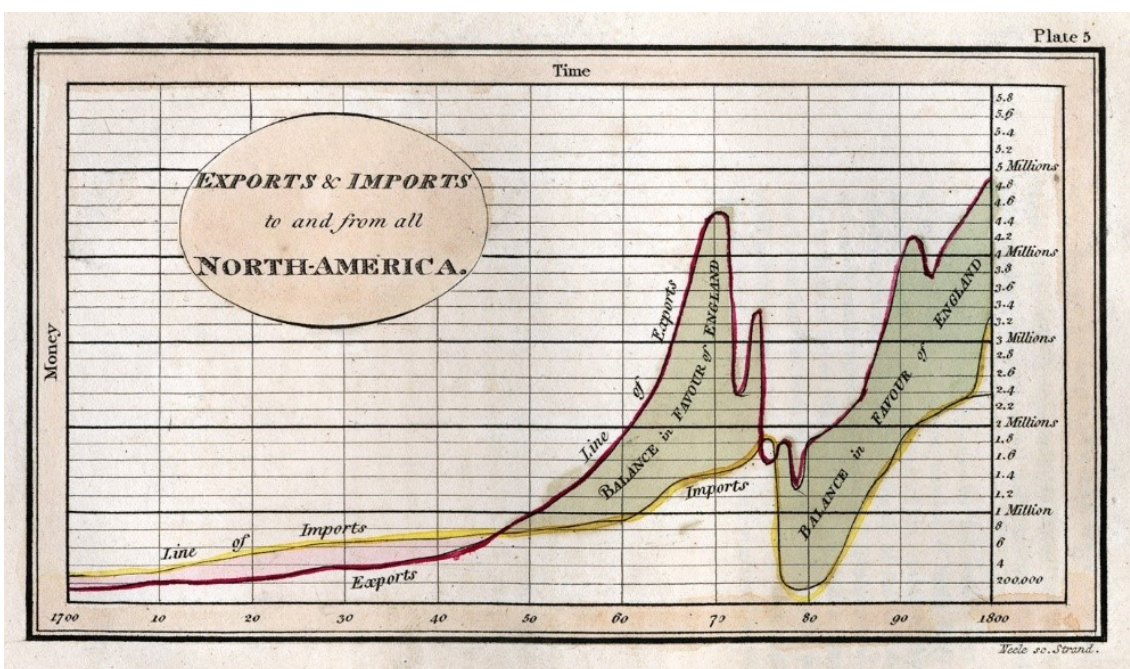

Fig. 3 : Line graph narrating the development of the British economy in the field of export-import to and from North America (reprint from [9])

subjectivity in the stages of visualization compilation. Subjectivity increases the attractiveness of visual appearance.

\section{Plot Visualization Using Chart}

Changes in quantity over time indicate growth. Relationships between variables can be observed from the journey of growth. This section explores visualization techniques to display variables that contain quantity values.

\section{A. History Chart}

Priestley visualizes the life history of famous figures in a line chart [3]. Initially, the data is represented in the form of a catalogue table (see Fig. 2), then the catalogue is visualized as a bar chart. Each line represents a character. The length of the line encodes the age of the character. Each age line is visualized as a bar. Each bar is arranged in a chronological dimension.

Fig. 1 illustrates pieces of succession to the kingdom of Jews. The chart tells the history of the life journey of royal leaders. The duration of the ruling figure can be observed visually. The reader cannot capture the relationships between the characters explicitly. The implied meaning that can be obtained by the reader is to have met each other or lived in almost the same period.

\section{B. Export-Import Chart}

Playfair visualized the history of British economic politics in the eighth century [9]. Playfair illustrates the export-import value with a line chart. The export-import line grows in the direction of time. Charts are displayed in a time dimension and sales volume space.
Exports and imports are coded in different colors. In [9], the export line is coded in red, and imports are coded in yellow. Playfail shades the area between the two lines. The color of the area tells the balance of export and import values. The surplus is coded in bright green, while the deficit is coded in bright red. The reader can find out the width of the gap between exports and imports easily.

Fig. 3 is an export-import chart narrating the growth of the British economy in the field of exports and imports from and to North America in $1700 \mathrm{AD}$ to $1800 \mathrm{M}$. The reader can easily identify periods of deficit and surplus. Readers can quickly see red or green areas. The critical point occurred in the early 1770s, where exports experienced a decline, while imports continued to grow even though they were less significant. Both countries' exports grew again in the early 1780s.

\section{Histomap}

Spark's Histomap narrates a history of four thousand years [10], [11]. Spark published his work in 1931. The graph shows a comparison of the relative strengths of nations, nations, and kingdoms. History begins in $2000 \mathrm{BC}$ to $2000 \mathrm{AD}$ The historical map is arranged vertically extends, see Fig. 4. History grows from top to bottom.

Each subject is coded with a colored channel. Maps form a flow of strength. The width of the channel shows the magnitude of strength. Based on the map content it can be seen that the forces compiled from activities that have a large impact on the subject. Some subjects are very similar in color, so the map needs to be read more thoroughly.

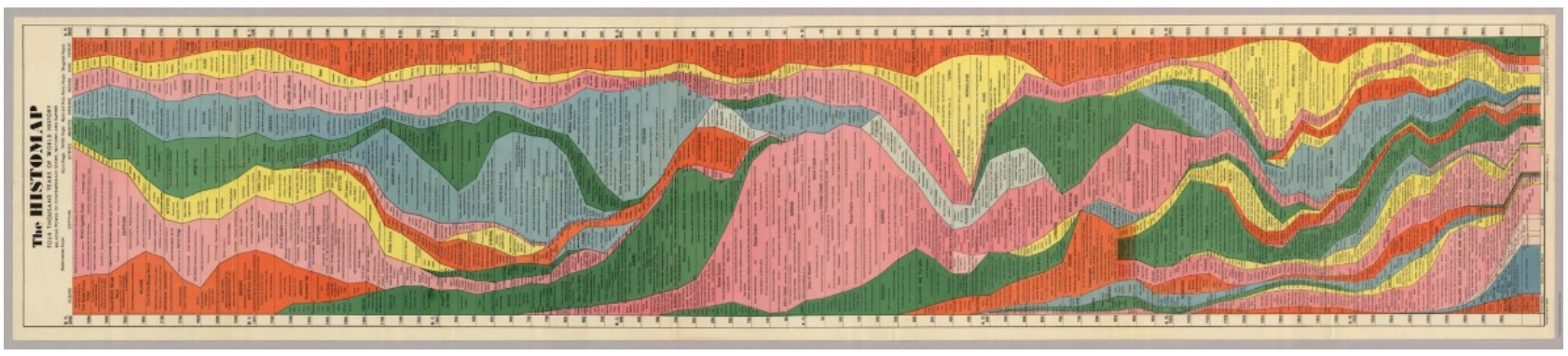

Fig. 4 : Histomap by Jhon B. Sparks, rotated 90 degrees to the left, (reprint from [11]). 


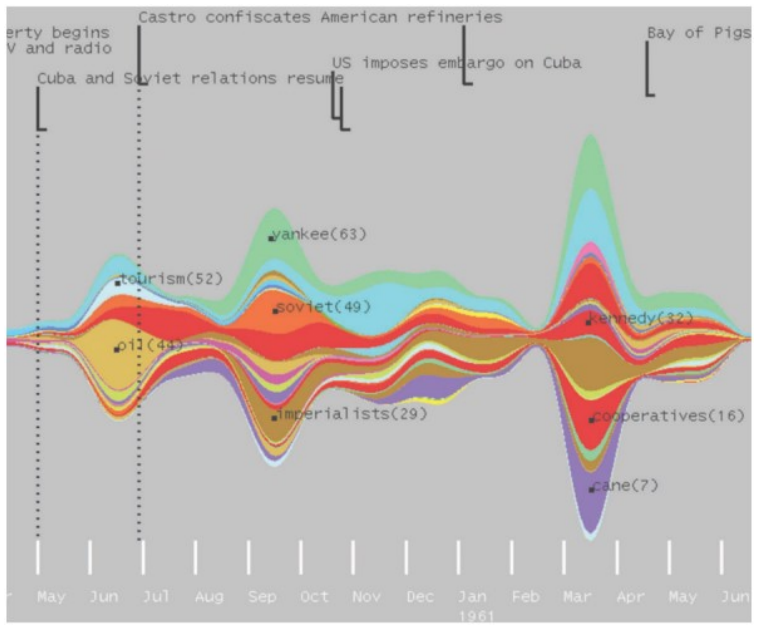

Fig. 5 : Theme River that narrate about the growth of topics from articles about Fidel Castro (reprint from [12]).

\section{Theme River}

Havre et al. visualize the development of themes from news articles using techniques such as histomap [12]. Flow strength describes the number of documents discussing a theme. Theme River development from the stacked bar chart. Havre et al. arrange the bar connected on the same topic so that the connectedness bar forms a flow of quantity of topics.

Fig. 5 is Theme River, which narrates the journey of the topic about Fidel Castro in the late 1960s to early 1962. The visual appearance of Theme River makes it easy to observe the development of a topic. The Theme River visualization is easier to read than if the same data is displayed with a stacked bar chart in Fig. 4.

\section{E. Peta Minard}

Minard made a graphic map of the journey of the French army to Russia in 1812 to 1813 [13], [14]. Graphics in the form of line charts. The chart shows the path of the French army. The line connects cities that the French army has traversed. The width of the line indicates the number of soldiers. Every one millimeter represents ten thousand people. Minard distinguished the path to and from Russia.

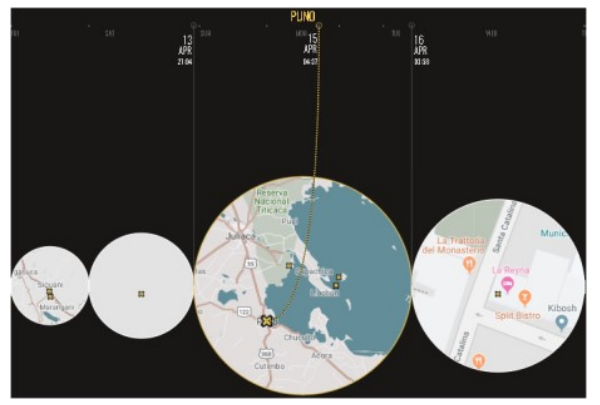

Fig. 7 : The Map Memento for Elice trip in Peru (reprint from [15]).

Departure and return routes are color coded. The Minard map depicts the journey with time implicitly

Fig. 6 narrates troop movements from the city of Kowno to Moscou (red line). The reader can see that the number of troops is decreasing drastically, as the troops approach Moscow. Troops only left thirty percent of the initial number departed. Returning troops are increasingly reduced (black line). The reader can guess the cause of repeated troop losses. Allegations can be linked to a chart of changes in air temperature located below the map.

\section{F. The Map Memento}

Memento visualization narrates a personal journey chronologically [15]. Visit locations are displayed in a circle. The circle shows a topographic map of the locations you've visited. The diameter indicates the duration (stay) of visits [16]. The topographic circles are arranged according to the time of occurrence. If a location is visited repeatedly, a circle appears more than once. The memento map tells about travel with explicit written time. Fig. 7 part of the Memento map that depicts cities Elice once visited during a trip to Peru.

\section{Plot Visualisation USING DiagRAM}

Interaction is one of the elements of the story. This section discusses the visualization of interactions between characters and between events. Interactions between characters are visualized by visualizing plots, metro maps, and story curves.

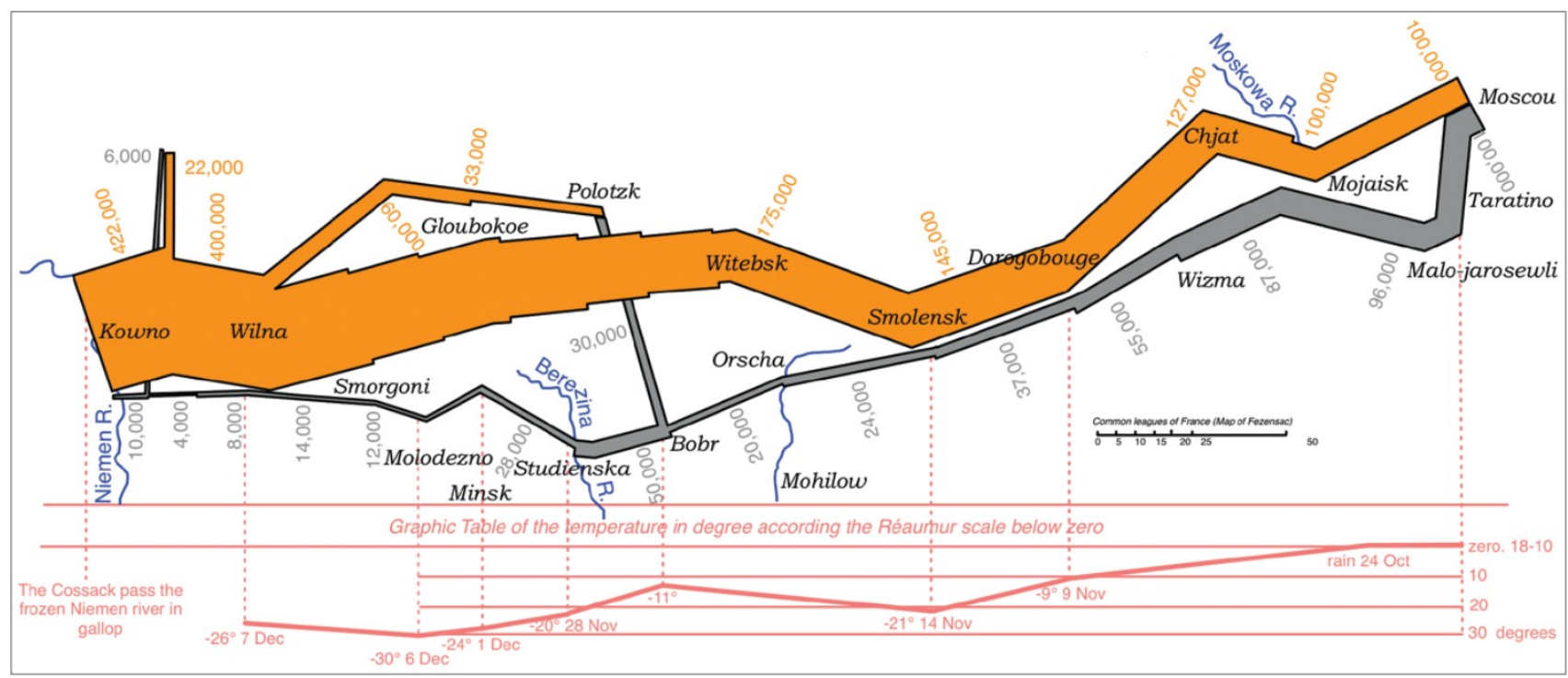

Fig. 6 : Map of French troop movements on the military operation in Russia in 1812-1813 (reprint from [13]) 


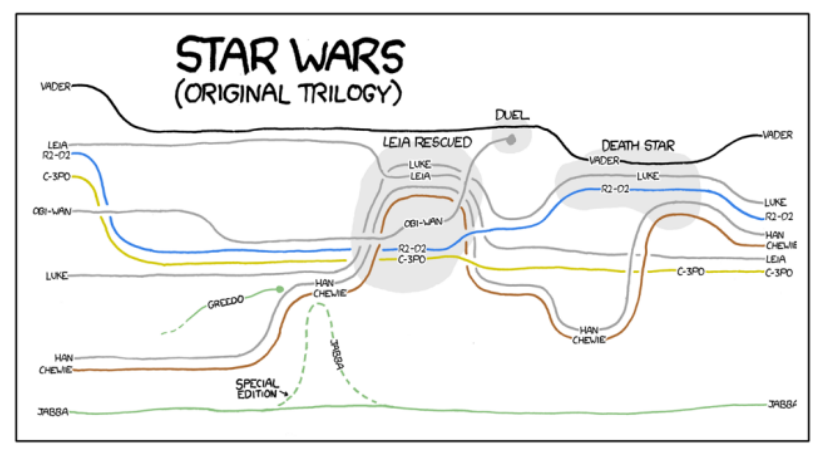

Fig. 8 : Fragment of Star Wars - Trilogy (Modification from [17]).

\section{A. Plot Visualisation}

The visualization plot was introduced by Munroe [17]. Munroe made a Movie Narrative Chart visualize the interaction between characters in the film. Munroe encodes the character with a line. The color of the line as the identity of each character. The lines that are close together indicate the characters who interact with each other. Shaded area indicates a battle or important event. This area is used to mark interesting interactions to be interpreted (such as conflicts).

Fig. 8 is a fragment of the film "Star Wars-Trilogy". The chart tells the interaction between the characters in the film. Putri Leia's rescue event was marked by a shaded area because it was an important event that needed to be highlighted to the reader. The duel between Vader and ObiWan is a critical event, where the event ends with the death of Obi-Wan, so it is marked by a shaded area. This diagram can help film analysts to see the story in the film quickly and quickly.

The Narrative Chart Movie is a handmade diagram. Ogievetsky developed a web application called Plotweaver [18]. The result is not neat. Between lines, there are still unnecessary crosses and turns, so the layout of the lines needs to be tidied up by reducing crosses and turns. The reduction of crossing becomes the focus of improvement. Some researchers reduce the crossing of a single line [19][22], and lines in a block [23].

Visualization of the plot has been applied to display interactions in various fields. Tapaswi et al. use plot visualization to display interactions in automatically detected movie scenes [24]. Ogawa uses plot visualization to narrate software development [25]. Kraak duplicates the Minard map to plot visualization form [13]. Kraak eliminates the number of troops and adds a flow of interaction between units.

\section{B. Metro Map}

Metro map is a diagrammatic topology map that illustrates public transportation routes [26], [27]. Initially, the

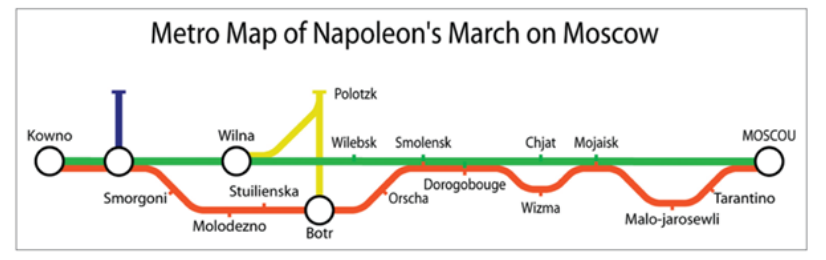

Fig. 9 : Minard Map has been represented in the form of a Metro Map (reprint from [13]).
Metro map was used to describe train routes. Then, the use of the metro map was expanded for public transportation. Metro map depicts routes with colored lines. The route is drawn by considering the location of the station and ignoring the road topology.

Kraak and Shahaf used the metro map to draw interaction maps [16], [19], [20]. Kraak modified the Minard map to form a metro map [16]. Kraak made two main routes (Fig. 9). The army route to Moscow, and the return route to Kowno. Kraak encodes a city that is passed by a stop or transit station. Shahaf uses the metro map to visualize relations between news articles. Stations symbolize documents and are sorted chronologically. The word is analogous to a train, and if a word is in a document, then the document is a stop.

\section{Story Curve}

Story Curve visualizes stories in dimensions and narrative dimensions [28]. The narrative dimension is timeoriented, while the story dimension is oriented in the order in which the real event appears. The narrative is parallel to the $x$-axis, and the story is parallel to the $y$-axis. Bars symbolize figures with color as identity. Each bar signifies the activities of a character.

Fig. 10 is the story curve narrating the Pulp Fiction film. Data sourced from conversational transcripts in the film. The sentence sequence of the transcript becomes the value of the position of the narrative. The sequence of real events becomes the position of the story. Real events are grouped into three parts (begin, middle, end).

\section{Shelleys Autobiography}

Shelley narrated his autobiography in the form of images [29]. Shelley describes the flow of events that she lived in from birth through 2006, see Fig. 11. The event contains facts. Shelley describes a fact as a point. The points are arranged by a curve so that it appears as a flow of facts in the event channel. Because of the limited visual media, Shelley only described the facts in the outline. Facts interact and often develop with time. The development of interactions is described in chronological space. The development of facts is in the space of the change of location. The order of the location of events develops with time and is drawn parallel to the $x$-axis.

Channels stream facts from the shortest time. The width of the channels dynamically develops in accordance with the number of facts that correspond to time. Two channels can be joined and branched. The entertainment and escape channels lead to the same channel. Channels of professional painters are formed from several channels, including entertainment, interests, and identities. Supporting facts are written around a series of main facts.

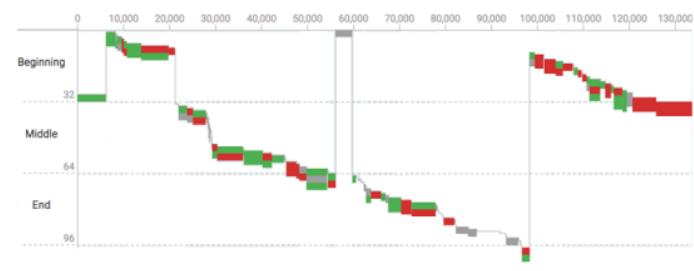

Fig. 10 : Story curve chart of Pulp Fiction (reprint from [21]). 


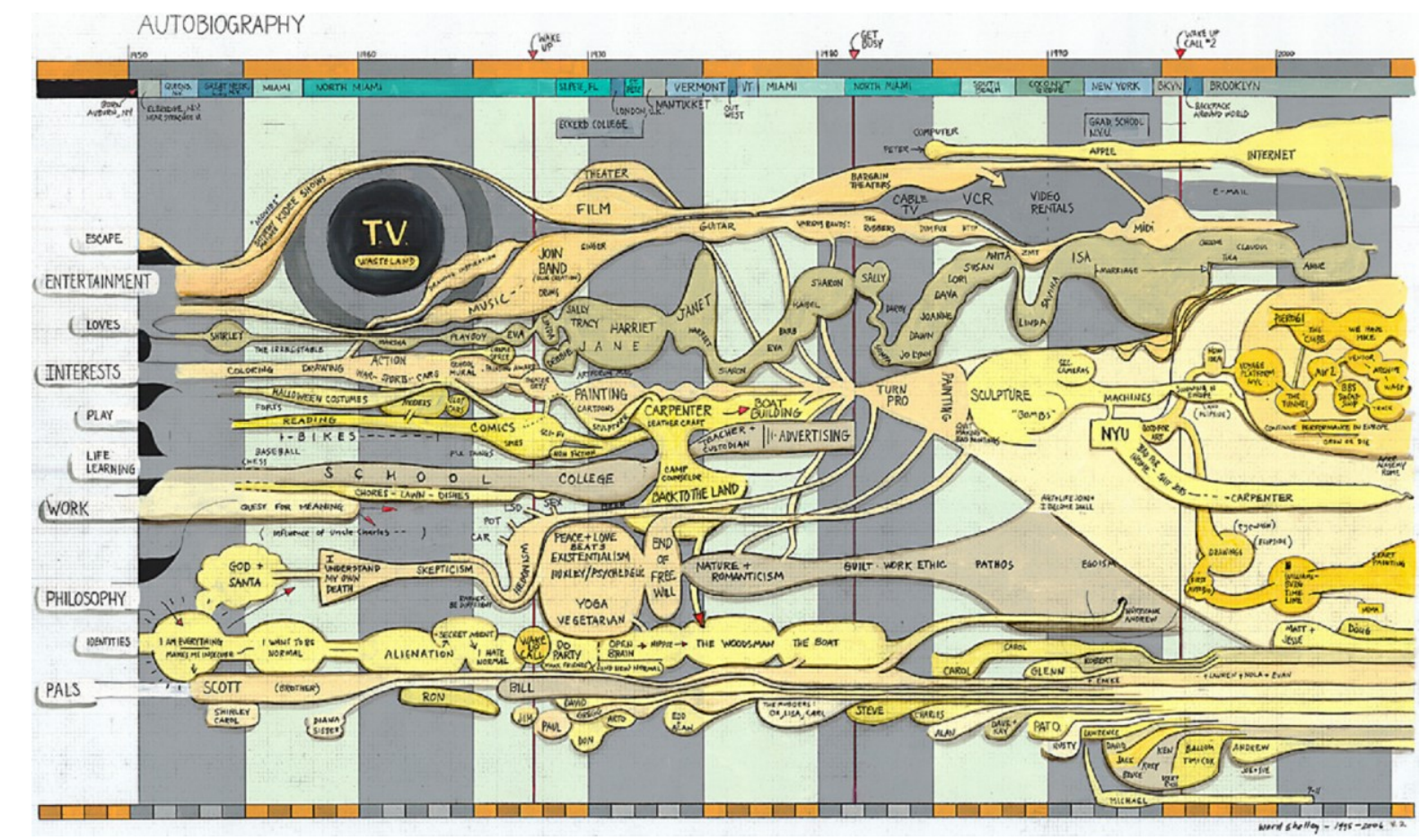

Fig. 11 : Autobiography Chart by Shelley (reprint from [29]).

\section{DISCUSSION}

This survey discusses the development of visualization techniques for displaying time-oriented data. The discussion takes precedence over the use of visualization to narrate the sequence of events. Visual models are grouped in charts and diagrams. Charts describe the flow of quantities and visits, while the diagram illustrates the flow of interactions. We identify the existence of story elements [30] from each visualization, see in table $I$, then arrange in a map the potential for completeness and flexibility, see in table II.

Historical and Export-Import charts are categorized in quantity flow. Both graphs show the change in the value of the entity. The history chart tells the life span of several figures. This visualization does not accommodate interactions between characters and the scene. The ExportImport chart narrates the travel of the export-import balance. Because exports and imports are conflicting entities, relations are in the area between the two. The subjectivity of the color of the shading in that area reinforces the meaning of surplus or deficit. This chart only applies to two entities.

TABLE I. EXISTENCE OF STORY COMPONENTS IN TIME-ORIENTED DATA VISUALIZATION

\begin{tabular}{|l|c|c|c|c|}
\hline \multicolumn{1}{|c|}{ Technic } & Event & Location & $\begin{array}{c}\text { Charact } \\
\text { er }\end{array}$ & Relation \\
\hline History chart & - & - & multi & implicit \\
\hline $\begin{array}{l}\text { Export-Import } \\
\text { Chart }\end{array}$ & gap & - & double & explicit \\
\hline Histomap & - & - & multi & implicit \\
\hline ThemeRiver & - & - & multi & implicit \\
\hline Minard Map & visit & geography & singgle & - \\
\hline Memento Map & visit & geography & singgle & - \\
\hline $\begin{array}{l}\text { Plot } \\
\text { Visualization }\end{array}$ & interaction & - & multi & explicit \\
\hline Metro Map & interaction & stasion & multi & explicit \\
\hline Story Curve & interaction & - & multi & implicit \\
\hline $\begin{array}{l}\text { Shelleys } \\
\text { Autobiography }\end{array}$ & fact & variable & singgle & explicit \\
\hline
\end{tabular}

TABLE II. MAP OF THE BENEFITS OF TIME-ORIENTED DATA VISUALIZATION FOR STORYTELLING

\begin{tabular}{|c|l|c|c|c|}
\hline \multicolumn{2}{|c}{ Potency } & \multicolumn{3}{|c|}{ Completeness } \\
\cline { 3 - 5 } & Low & Middle & High \\
\hline \multirow{2}{*}{ Low } & M03, M04 & M02, M05 & - \\
\cline { 2 - 5 } \\
\cline { 2 - 5 } & Middle & M01 & M06, M09 & M07,M08, \\
\cline { 2 - 5 } & High & - & - & - \\
\cline { 2 - 5 } & & - & - & M10 \\
\hline
\end{tabular}

M01 : History Chart, M02 : Export-Import Chart, M03 : Histomap, M04 : ThemeRiver, M05 : Minard Map, M06 : Memento Map, M07 : Plot Visualization, M08 : Metro Map, M09 : Story Curve, M10 : Shelleys Autobiography.

The Export-Import chart is fixed with the Histomap and Theme River chart. Histomap and ThemeRiver can be used to display multiple entities. The use of both is specific to entities with the same topic. The area shows the difference in strength between entities. The meaning of difference needs to be interpreted by the reader himself.

Minard map is the trajectory of the visit above the topographic map. The width of the path shows changing characters, while the color indicates the direction. Time appears implicitly as the duration of a trip from one place to another. The Memento map is similar to the Minard map, but the Memento map compiles a chronological visit map. This spatial flow map does not show relations. This map is only suitable for single characters.

Visualization in the interaction flow category displays the flow of connectivity between characters. Metro Map and Shelley's Biography can observe the location of events from a visual display. Shelley becomes the location as an event variable. Locations in the Visualization plot can be seen as equivalent to the event. Story curves cannot be directly observed from the graph location. Visualization in this category narrates the activities of several characters. 
Especially for Shelley's Autobiography, placing supporting figures as passive figures. The visualization plot and the metro map visualize interactions with the intersection of lines, while Shelley's Biography is visualized with points of fact with names. Especially for the story curve, relations do not appear explicitly.

Apart from the completeness of story elements, we examine the benefits of data visualization as a medium for storytelling. We map visualization techniques based on flexibility and completeness (table II). The map contains nine quadrants. Each potential is given a low, middle, and high value. Flexibility is the ability of graphics to be summarized or detailed using the same visual model. Flexibility is judged by the ability to scale, the direction of flow, and the completeness of the content. Completeness is derived from the existence of story components in visualization.

Almost all charts have low flexibility. The flexibility and completeness of Histomap and ThemeRiver are very low. The export chart and Minard Map are in an area with low flexibility, while the completeness is at the middle level. The History chart is more flexible than the four charts above, but the completeness is very low. Memento Map is a good chart because both of its potentials are at the middle level.

Diagrams are a good enough medium for storytelling. The four diagrams are in the middle flexibility quadrant. The Story Curve is at the intermediate level. Its completeness and flexibility are at the intermediate level. Plot Visualization, Metro Map, and Shelleys Autobiography have high levels of completeness with flexibility at the intermediate level.

\section{CONCLUSION}

Visualization of time-oriented data has grown from the visualization of changes in quantity to the visualization of interactions. It shows that visualization in the time dimension can be used to tell a story. Because graphics are based on data, this visualization is very suitable for telling non-fiction stories. However, this visualization needs to develop visual techniques to be able to accommodate the completeness of story elements and shape flexibility. It should also be noted that the visualization of the five $\mathrm{W}$ elements in data or facts.

\section{ACKNOWLEDGMENT}

This work was supported by the Ministry of Religion of the Republic of Indonesia and the Ministry of Education and Culture of the Republic of Indonesia.

\section{REFERENCES}

[1] E. Segel and J. Heer, "Narrative visualization: Telling stories with data," IEEE Trans. Vis. Comput. Graph., vol. 16, no. 6, pp. 11391148, 2010.

[2] A. Rosenber, Daniel; Grafton, Cartographies of Time: a History of the Timeline. 2010.

[3] J. Priestley, A Description of a New Chart of History: Containing a View of the Principal Revolutions of Empire that Have Taken Place in the World, 6th ed. London: J. Johnson, 1786.

[4] S. Liu, W. Cui, Y. Wu, and M. Liu, "A survey on information visualization: recent advances and challenges," Vis. Comput., vol. 30, no. 12, pp. 1373-1393, Dec. 2014.

[5] Merriam-Webster, "Definition of visualization," Merriam-Webster, 2019. [Online]. Available: https://www.merriam-webster.com [Accessed: 07-Oct-2019].
[6] C. Chen, W. Härdle, A. Unwin, and M. Friendly, "A Brief History of Data Visualization,” Handb. Data Vis., pp. 15-56, 2008.

[7] S. F. Silva and T. Catarci, "Visualization of linear time-oriented data: A survey," Proc. 1st Int. Conf. Web Inf. Syst. Eng. WISE 2000, vol. 1, no. March, pp. 310-319, 2000.

[8] A. Thudt, C. Perin, W. Willett, and S. Carpendale, "Subjectivity in personal storytelling with visualization,” Inf. Des. J., vol. 23, no. 1, pp. 48-64, 2017.

[9] W. Playfair, The Commercial and Political Atlas: Representing, by Means of Stained Copper-plate Charts, the Progress of the Commerce, Revenues, Expenditure and Debts of England During the Whole of the Eighteenth Century. T. Burton, 1801.

[10] J. B. Sparks, Histomap of World History, Illustrate. Rand McNally, 1990.

[11] N. Routley, "Histomap: Visualizing the 4,000 Year History of Global Power," $2017 . \quad$ [Online]. Available: www.visualcapitalist.com/histomap/. [Accessed: 11-Nov-2019].

[12] S. Havre, I. C. Society, E. Hetzler, P. Whitney, and L. Nowell, "ThemeRiver: Visualizing Thematic Changes in Large Document Collections," vol. 8, no. 1, pp. 9-20, 2002.

[13] M. Kraak, Mapping Time: Illustrated by Minard's Map of Napoleon's Russian Campaign of 1812, Illustrate. Redlands: Esri Press, 2014.

[14] M. Friendly, "Visions and Re-Visions of Charles Joseph Minard," J. Educ. Behav. Stat., vol. 27, no. 1, pp. 31-51, Mar. 2002.

[15] A. Thudt, D. Baur, S. Huron, and S. Carpendale, "Visual Mementos: Reflecting Memories with Personal Data," IEEE Trans. Vis. Comput. Graph., vol. 22, no. 1, pp. 369-378, 2016.

[16] A. Thudt, D. Baur, and S. Carpendale, "Visits : A Spatiotemporal Visualization of Location Histories," in Eurographics Conference on Visualization, 2013.

[17] Randall Munroe, "Movie Narrative Charts," Xkcd, 2009. [Online]. Available: http://xkcd.com/657/. [Accessed: 07-Jun-2017].

[18] V. Ogievetsky, "Plotweaver," Computer Science Department of Stanford University, 2009. [Online]. Available: graphics.stanford.edu/wikis/cs448b-09-fall/FP-OgievetskyVadim.

[19] Y. Tanahashi and K. L. Ma, "Design considerations for optimizing storyline visualizations," IEEE Trans. Vis. Comput. Graph., vol. 18, no. 12, pp. 2679-2688, 2012.

[20] S. Liu, Y. Wu, E. Wei, M. Liu, and Y. Liu, "StoryFlow: Tracking the Evolution of Stories," IEEE Trans. Vis. Comput. Graph., vol. 19, no. 12, pp. 2436-2445, Dec. 2013.

[21] Y. Tanahashi, C. H. Hsueh, and K. L. Ma, "An efficient framework for generating storyline visualizations from streaming data," IEEE Trans. Vis. Comput. Graph., vol. 21, no. 6, pp. 730-742, 2015.

[22] M. Gronemann, M. Jünger, F. Liers, and F. Mambelli, "Crossing minimization in storyline visualization," in Lecture Notes in Computer Science, 2016, vol. 9801 LNCS, pp. 367-381.

[23] T. C. Van Dijk et al., "Block crossings in storyline visualizations," in Lecture Notes in Computer Science, 2016, vol. 9801 LNCS, pp. 382398.

[24] M. Tapaswi, M. Bauml, and R. Stiefelhagen, "StoryGraphs: Visualizing Character Interactions as a Timeline," in 2014 IEEE Conference on Computer Vision and Pattern Recognition, 2014, pp. 827-834.

[25] M. Ogawa and K.-L. K. Ma, "Software evolution storylines," SOFTVIS '10 Proc. 5th Int. Symp. Softw. Vis., pp. 35-42, 2010.

[26] A. Cain, Designing Printed Transit Information Materials: A Guidebook for Transit Service Providers. Tampa, FLorida, 2008.

[27] P. Bain, “Aspects of Transit Map Design,” Parsons J. Inf. Mapp., vol. 2, no. 3, 2010.

[28] N. W. Kim, B. Bach, H. Im, S. Schriber, M. Gross, and H. Pfister, "Visualizing Nonlinear Narratives with Story Curves," IEEE Trans. Vis. Comput. Graph., vol. 24, no. 1, pp. 595-604, 2018.

[29] W. Shelley, “Autobiography Version 2," www.wardshelley.com, 2006. [Online]. [Accessed: 26-Oct-2019].

[30] R. McKee, Story: Style, Structure, Substance, and the Principles of Screenwriting. New York, USA: Harper Collins, 1997. 